\title{
How to Make a Kin Selection Model
}

\author{
Peter D. Taylor $\dagger$ and Steven A. Frank $\ddagger$ \\ $\uparrow$ Department of Mathematics and Statistics, Queen's University, Kingston, OT. K7L 3N6, and \\ $\$$ Department of Ecology and Evolutionary Biology, University of California, Irvine, CA 92717, \\ U.S.A.
}

(Received on 24 July 1995, Accepted in revised form on 8 December 1995)

\begin{abstract}
Kin selection arguments, based on Hamilton's (1964) concept of inclusive fitness, provide a powerful heuristic and can therefore give us valuable insights into the different pathways through which natural selection acts. But their formulation can be quite tricky, requiring as they do, a close accounting of all the fitness effects of a particular item of behaviour. Here we propose a "direct fitness" formulation of inclusive fitness which often has a more straightforward derivation. Our method finds ESS trait values by the standard optimization techniques of simple differentiation plus two additional steps. First, slopes of group phenotype on individual genotype arise naturally during differentiation, and these slopes are replaced by coefficients of relatedness. Second, when behaviours influence different classes such as age, sex of recipient, or other life history components of fitness, the fitness effects on each component are weighted by reproductive value. We illustrate this technique first in a homogeneous population, with examples of group competition and partial dispersal behaviour, and then in a class-structured population, with examples of sex allocation and altruism between age classes.
\end{abstract}

(C) 1996 Academic Press Limited

\section{Introduction}

Kin selection is widely accepted as a fundamental process of evolution. The standard tool for modelling kin selection is the technique of inclusive fitness introduced by Hamilton (1964). Because kin selection arguments focus on social behaviours and their reproductive consequences for different individuals, the equations that arise in this modelling approach can give us valuable insights into the different pathways through which natural selection acts. And it is known that under mildly restrictive assumptions (e.g. weak selection and additive gene action) this approach will give us the same ESS as the more complex and less revealing one-locus genetic model (Hamilton, 1975; Charnov, 1977; Charlesworth, 1980; Michod \& Hamilton, 1980; Seger, 1981; Grafen, 1985, Taylor, 1989, 1990).

With such power, both conceptual and technical, it is not surprising that the inclusive fitness method, requiring as it does a close accounting of all the fitness effects of a particular item of behaviour, is often difficult to formulate. Our purpose here is to propose a "direct fitness" approach which will always produce an expression for the inclusive fitness of an actor, but often with a more straightforward derivation. We illustrate this result with a number of examples, first in a homogeneous population, and then in a class-structured population; in the latter case it is necessary to pay attention to reproductive value.

\section{Homogeneous Population}

A simple general example will illustrate this method. We consider a population in which a variable $x$ determines individual behaviour (sex allocation, dispersal rate, level of altruism, etc.), and we seek an evolutionarily stable level $x^{*}$ of that behaviour in the population. The standard ESS condition (Maynard Smith \& Price, 1973) is that an individual whose behaviour deviates from $x^{*}$ should have reduced fitness:

$$
W(x) \leqslant W\left(x^{*}\right)
$$


If this holds for all $x$ near $x^{*}$, then $\mathrm{d} W / \mathrm{d} x$ will be zero at $x^{*}$, and this gives us an equation to solve for $x^{*}$ :

$$
\left.\frac{\mathrm{d} W}{\mathrm{~d} x}\right|_{x=x^{*}}=0
$$

An important complication is that fitness $W$ will typically depend not only on individual behaviour, but on the behaviour of neighbours, and a deviant individual is apt to have deviant neighbours. This complication is generally thought to make the differentiation method for finding ESSs unworkable for interactions among kin without resort to special techniques such as the Price (1970) equation.

We show that interactions among relatives can be handled with the standard differentiation approach by using the following set-up. We distinguish two kinds of individuals: "actors," whose behaviour is correlated with their genotype, and "recipients," whose fitness is affected by the behaviour of one or more actors. An individual may play both roles. Genes work through actors (genotype affects phenotype) but gene frequencies change through fitness effects on recipients. Our approach will be to take a monomorphic population, that is, a population with a constant genic value $x^{*}$ at the focal locus. We then select a random allele at the behavioural locus, mutate that allele and its IBD copies to a deviant value $x$, and ask how the fitness $W$ of its bearer changes in response. The point is that neighbouring actors who are related may also have the allele, and will experience a change in behaviour, and this will alter the fitness of the recipient. The equilibrium condition for $x^{*}$ will be that $\mathrm{d} W / \mathrm{d} x=0$ at $x=x^{*}$.

To take a simple concrete example, suppose the allele resides in a female whose fitness $W$ depends on her own phenotype $y$ and on the average phenotype $z$ of a group of neighbours:

$$
W=W(y, z) .
$$

Now we ask how $W$ changes with $x$. If we calculate the derivative of $W$, using the chain rule, we get:

$$
\frac{\mathrm{d} W}{\mathrm{~d} x}=\frac{\partial W}{\partial y} \frac{\mathrm{d} y}{\mathrm{~d} x}+\frac{\partial W}{\mathrm{~d} z} \frac{\mathrm{d} z}{\mathrm{~d} x} .
$$

It is not clear what sense to make of the phenotypic derivatives as $y$ and $z$ are not strictly functions of $x$. However, $\mathrm{d} y / \mathrm{d} x$ and $\mathrm{d} z / \mathrm{d} x$ are slopes of actor phenotype on genic value, and if we replace them by the corresponding statistical regression coefficients, we get

$$
\frac{\mathrm{d} W}{\mathrm{~d} x}=\frac{\partial W}{\partial y} \beta_{y x}+\frac{\partial W}{\partial z} \beta_{z x} .
$$

If we divide (2) by $\beta_{y x}$, we get a formulation using coefficients of relatedness which turns out (Table 1) to be exactly the inclusive fitness effect $\Delta W_{I F}$ of a random actor-more precisely, the rate of change of the inclusive fitness with $x$ :

$$
\Delta W_{I F}=\frac{\partial W}{\partial y}+\frac{\partial W}{\partial z} R
$$

where

$$
R=\frac{\operatorname{cov}(z x)}{\operatorname{cov}(y, x)}
$$

is the relatedness of a female $y$ to a random neighbour $z$. This is the personal fitness formulation of relatedness proposed by Orlove \& Wood (1978), but it is equivalent to the inclusive fitness form of Michod \& Hamilton (1980), as pointed out by Queller (1985). The replacement of the phenotypic derivatives (which are evaluated at the equilibrium $x^{*}$ ) with the regression coefficients is reasonable if the population variance is small, that is, if the mutation has small effect. If the variance of $x$ is appreciable, eqn (2) still bears a close relationship to Queller's (1992) formulation of Price's (1970) equation; this correspondence is explored in Appendix B.

This result shows that the standard maximization method for solving ESS problems can be adapted to analyse models with interactions among relatives - if we simply replace phenotypic derivatives by the corresponding relatedness coefficients, $\mathrm{d} W / \mathrm{d} x$ will turn into the inclusive fitness of a random actor. The method is illustrated with two examples.

TABLE 1

The inclusive fitness calculation

\begin{tabular}{lccc}
\hline Recipient & Number & Relatedness & Fitness change \\
\hline Self & 1 & 1 & $\frac{\partial W}{\partial y} \delta$ \\
Neighbour & $n$ & $R$ & $\frac{\partial W}{\partial z} \frac{\delta}{n}$ \\
\hline
\end{tabular}

$$
\Delta W_{I F}=\mathrm{SUM}=\delta\left[1 \frac{\partial W}{\partial y}+n R \frac{\partial W}{\partial \mathrm{z}} \frac{1}{n}\right]=\delta\left[\frac{\partial W}{\partial y}+R \frac{\partial W}{\partial z}\right] .
$$

Consider a female (the actor) who increases her phenotypic value $y$ by $\delta$. The inclusive fitness method requires us to add up the fitness effects of this deviation on all neighbouring individuals (including herself), each effect weighted by the relatedness of the affected individual to the actor. In this case, there is her direct effect on her own fitness, and her effect on the fitness of each of the $n$ neighbours in the group, which acts through the change in $y$ of $\delta / n$. The expression in the square brackets is exactly formula (3). 
EXAMPLE 1

\section{Protocells and virulence-competitiveness in a group}

A common fitness trade-off concerns the tension between competition among neighbours for limited resources and the success of the neighbourhood against other groups. One example of this is the competition among related copies of a replicator molecule within primitive cells (protocells). A replicator gains by outcompeting its neighbours within the cell, but overuse of local resources may slow cell division and reduce overall genetic contribution to the population. Several authors have noted that this is a kin selection problem (Maynard Smith, 1979; Bresch et al., 1980; Szathmary \& Demeter, 1987). The more closely related the replicators within a cell, the less they will compete and the more they will promote the overall success of the entire cell.

The protocell problem is analogous to certain aspects of parasite evolution. When there are different parasite genotypes within the host, there is competition for host resources. That may lead to higher rates of replication within the host, causing increased damage to the host (virulence). However, reducing host vigor may reduce the resources available to the parasites, thus reducing the overall genetic contribution of the parasites to the population (Hamilton, 1972; Bremermann \& Pickering, 1983).

The simplest example capturing this tension between within-group competition and prudent use of local resources is

$$
W=W(y, z)=\frac{y}{z} \cdot G(z)
$$

where $y$ sets the competitiveness of an individual with the $x$-gene, and $z$ is the average value of $y$ within the individual's group. Here, $G(z)$ measures the resources available to the group, and is supposed to be a decreasing function of average group character $z$. Observe that $G(z)$ is the average fitness in a $z$-group.

The derivative of $W$ is

$$
\frac{\mathrm{d} W}{\mathrm{~d} x}=\frac{1}{z} \cdot G(z) \frac{\mathrm{d} y}{\mathrm{~d} x}+\left[-\frac{y}{z^{2}} \cdot G(z)+\frac{y}{z} \cdot G^{\prime}(z)\right] \frac{\mathrm{d} z}{\mathrm{~d} x}
$$

If we evaluate this at $y=z=y^{*}$ and replace the phenotypic derivatives by the corresponding relatedness coefficients we get

$$
\Delta W_{I F}=\frac{1}{y^{*}} \cdot G+R\left[-\frac{1}{y^{*}} \cdot G+G^{\prime}\right]
$$

where $R$ is the relatedness of an actor to a random member of its group (including itself), $G$ and $G^{\prime}$ are evaluated at $y^{*}$. Referring to (3), the first term of (5) is $\partial W / \partial y$, and measures the gain to the actor of its increased competitiveness. The term in the square brackets of (5) is $\partial W / \partial z$, and it displays the two ways in which increased individual competitiveness reduces the resources available to the group - first, the effect of the increased share of the actor of a fixed total, and second, the effect of the increase in competitiveness which reduces the total resources available (note that $G^{\prime}$ is negative).

The simplest model for $G$ is $G(z)=1-c z$, where $c$ is the rate at which average group competitiveness reduces available resources. If we put this into (5), and set the expression to zero, we get the equilibrium to be (Frank 1994)

$$
c y^{*}=1-R
$$

\section{EXAMPLE 2}

\section{Partial dispersal of offspring}

Consider a haploid asexual population with $N$ females breeding on a patch, each of whom has a large number $n$ of offspring. Suppose a fraction $y$ of the offspring disperse and that a proportion $1-c$ of these find their way to a random patch in the population, so that $c$ is the cost of dispersal. We assume the dispersal rate $y$ is under maternal control. This is a generalization of Hamilton \& May's 1977 dispersal model.

The fitness of a mother who disperses offspring at rate $y$ on a patch with average rate $z$ in a population with normal rate $y^{*}$ is:

$$
W(y, z)=n(1-y) p(z)+n y(1-c) p\left(y^{*}\right)
$$

where

$$
p(z)=\frac{1}{n\left(1-z+y^{*}(1-c)\right)} .
$$

is the probability an offspring competing on a $z$-patch will win a breeding spot.

The derivative of $W$ evaluated at $y^{*}$ is

$$
\begin{aligned}
\frac{\mathrm{d} W}{\mathrm{~d} x}=\frac{\partial W}{\partial y} & \frac{\mathrm{d} y}{\mathrm{~d} x}+\frac{\partial W}{\partial z} \frac{\mathrm{d} z}{\mathrm{~d} x} \\
& =-n p+n(1-c) p+n\left(1-y^{*}\right) p^{\prime} \frac{\mathrm{d} z}{\mathrm{~d} x}
\end{aligned}
$$

If we simplify and replace the derivative by the relatedness $R$ of a female to her patchmates, we get

$$
\Delta W_{I F}=\frac{1}{1-y^{*} c}[-c+R k] .
$$


where

$$
k=\frac{1-y^{*}}{1-y^{*} c}
$$

is the probability a random breeding female is native to her patch.

Note that the expression in the square brackets of (8) gives the inclusive fitness gain of a female who disperses an extra offspring and retains one less at home. If our units of fitness are offspring ready to compete for a breeding spot, then the return through a disperser is $1-c$, and the return from a domestic is $1-R k$, where $R k$ is her expected relatedness to a random offspring competing on her home patch. This is an example of the accounting of competitive effects that is an essential and tricky aspect inclusive fitness arguments [Taylor 1992; see also eqns (22-24) below].

We calculate the ESS value of $y^{*}$ by setting (8) equal to zero, yielding

$$
y^{*}=\frac{R-c}{R-c^{2}}
$$

but we still have work to do as $R$ depends on $y^{*}$ and its value is calculated with a recursion equation (Frank, 1986a; Taylor, 1988a). When the resulting expression is put into (10), and the equation is solved for $y^{*}$, we get the equilibrium dispersal rate to be

$$
y^{*}=\frac{H+1-2 N c}{H+1-2 N c^{2}}
$$

where

$$
H=\sqrt{1+4 N(N-1) c^{2}} .
$$

\section{Class-structured Population}

We now turn to more complex problems to illustrate the full power of the method. These "class-based" models extend the notion of relatedness as a derivative and address the complications that arise when different kinds of individuals interact. Suppose there are different classes of individuals (e.g. by sex, by age, by size, by habitat, or some combination). If the behaviour of an actor affects the fitness of individuals in more than one class, we have the problem of comparing reproductive contributions between classes. The key concept here is that of reproductive value (RV) (Fisher, 1958; Taylor, 1990). We define: $c_{j}$ : the class RV, defined as the probability that the ancestor of a random gene in the distant future resides in a class $j$ individual today; $u_{j}$ : the frequency of class $j$ in the population; $v_{j}$ : the RV of an individual in class $j$. There are different ways of normalizing the $v_{j}$, but a convenient general formula is:

$$
v_{j}=c_{j} / u_{j} .
$$

Another normalization, commonly used in agestructured populations, sets the reproductive value $v_{0}$ of a zygote to be unity. These definitions are meant to apply to a monomorphic population (uniform $x^{*}$ ) at class-frequency equilibrium (constant $u_{j}$ ).

It is important to distinguish the $c_{j}$ from the $v_{j}$. For example, if the classes are the two sexes, male $m$ and female $f$, then in a haplodiploid population, a gene in the distant future has twice the probability of being in a female today as in a male today (Price, 1970) so that $c_{f}=2 / 3$ and $c_{m}=1 / 3$. On the other hand, when we are working with age classes, we typically use the $v_{j}$ defined in 1930 by Fisher (1958, p. 27). In inclusive fitness arguments, the $v_{j}$ are used as relative weights to compare additive fitness benefits to individuals of different classes.

How do we choose a random allele in a class-structured population? It turns out that the correct way to do this is to take it to be in a class- $j$ individual with probability $c_{j}$ so that its average fitness is

$$
W=\sum c_{j} W_{j}
$$

where $W_{j}$ is the fitness of a random class- $j$ individual. (The $c_{j}$ arise here because what we actually want to maximize is the asymptotic growth rate of the population of descendants of the allele. This formulation is justified in Appendix A.) Equation (13) assumes that the different $W_{j}$ are identically normalized - that is, they have the same value in the monomorphic $x^{*}$ population. In practice, the sum in (13) need only be over the recipient classes. We then calculate the derivative of $W$ with respect to the genic value $x$ of the gene, and the analysis proceeds as in the homogeneous case.

We will analyse the simplest case, in which the actors all belong to a single class but there may be recipients in a number of classes, including the actor class. Put another way, all phenotypic effects of varying $x$ occur to members of one class, the actor class. In case a gene affects behaviour of more than one class of actor, we suppose there are modifiers present which allow different responses in different classes, and we can do the analysis as a sequence of one-class problems. An important special case of this occurs when an actor's behaviour may depend conditionally on its state (size, stage of development, habitat etc.) and this can be handled by treating actors in different states as belonging to different classes.

We illustrate the calculations with a general 
example-one that is rich enough to completely describe the method and to demonstrate the equivalence of this approach with the standard inclusive fitness method. Suppose there are three classes: (1) juvenile males, (2) juvenile females, and (3) adult females. Suppose the actors are the adult females, and all three classes are recipients. Specifically, an actor can affect the fitness of (1) sons and nephews, (2) daughters and nieces, and (3) herself, and her sisters. (We use pedigree relationships here because it gives us convenient names, but the method applies to any pattern of relationships, for example, those determined by spatial proximity.) Thus, the class fitnesses have the functional form:

$$
\begin{array}{ll}
\text { class 1: } & W_{1}=W_{1}\left(y_{1}, z_{1}\right) \\
\text { class 2: } & W_{2}=W_{2}\left(y_{2}, z_{2}\right) \\
\text { class 3: } & W_{3}=W_{3}\left(y_{3}, z_{3}\right)
\end{array}
$$

where each of the variables is the average phenotype of an actor or group of actors:

$$
\begin{aligned}
& y_{1}=\text { the mother of the juvenile male } \\
& z_{1}=\text { the aunts of the juvenile male } \\
& y_{2}=\text { the mother of the juvenile female } \\
& z_{2}=\text { the aunts of the juvenile female } \\
& y_{3}=\text { the adult female herself } \\
& z_{3}=\text { the sisters of the adult female. }
\end{aligned}
$$

We have subscripted the variables because of the possibility of asymmetric relatedness coefficients between the sexes. For example, one might suppose that the common variable $y$ could be used for both $y_{1}$ and $y_{2}$, but we will want to replace $\mathrm{d} y_{1} / \mathrm{d} x$ and $\mathrm{d} y_{2} / \mathrm{d} x$ by the relatednesses of a female actor to a son and daughter respectively, and under an asymmetric genetic system such as haplodiploidy, these might be different. Thus the subscripts can serve as a guide to the correct relatedness coefficient.

According to (13) the average recipient fitness is

$$
W=c_{1} W\left(y_{1}, z_{1}\right)+c_{2} W_{2}\left(y_{2}, z_{2}\right)+c_{3} W_{3}\left(y_{3}, z_{3}\right)
$$

The derivative of $W$ is:

$$
\frac{\mathrm{d} W}{\mathrm{~d} x}=c_{1} \frac{\mathrm{d} W_{1}}{\mathrm{~d} x}+c_{2} \frac{\mathrm{d} W_{2}}{\mathrm{~d} x}+c_{3} \frac{\mathrm{d} W_{3}}{\mathrm{~d} x} .
$$

Note that in the differentiation, we can treat the $c_{j}$ as constants (calculated in the monomorphic $x^{*}$-population). This matter is discussed further in Appendix A.

We now show that, with the appropriate relatedness coefficients, eqn (15) gives us the inclusive fitness effect $\Delta W_{I F}$ of an adult female (Table 2). For example, the first term is
TABLE 2

The inclusive fitness effect of an adult female actor

\begin{tabular}{lcccc}
\hline Recipients & Number & Effect & Relatedness & RV \\
\hline Class 1 & & & & \\
$\quad$ sons & $n_{1}$ & $a_{1}$ & $r_{1}$ & $v_{1}$ \\
$\quad$ nephews & $m_{1}$ & $b_{1}$ & $R_{1}$ & $v_{1}$ \\
Class 2 & & & & \\
$\quad$ daughters & $n_{2}$ & $a_{2}$ & $r_{2}$ & $v_{2}$ \\
$\quad$ nieces & $m_{2}$ & $b_{2}$ & $R_{2}$ & $v_{2}$ \\
Class 3 & & & & \\
$\quad$ self & 1 & $a_{3}$ & 1 & $v_{3}$ \\
$\quad$ sisters & $m_{3}$ & $b_{3}$ & $R_{3}$ & $v_{3}$ \\
\hline
\end{tabular}

The second column lists the numbers of recipients of each type of a single actor. The effects in the third column are the rates at which recipient fitness increases with changes in the actor phenotype where we normalize fitness to unity. Thus, $a_{1}$ is the rate at which a male's fitness changes with respect to his mother's phenotype. The inclusive fitness effect is the sum of the effects on different recipients:

$$
\begin{aligned}
& \Delta W_{I F}=v_{1}\left[n_{1} r_{1} a_{1}+m_{1} R_{1} b_{1}\right]+v_{2}\left[n_{2} r_{2} a_{2}+m_{2} R_{2} b_{2}\right] \\
& +v_{3}\left[a_{3}+m_{3} R_{3} b_{3}\right] \\
& \begin{aligned}
p c_{1} \frac{\mathrm{d} W_{1}}{\mathrm{~d} x}= & c_{1}\left[\frac{\partial W_{1}}{\partial y_{1}} \frac{\mathrm{d} y_{1}}{\mathrm{~d} x}+\frac{\partial W_{1}}{\partial z_{1}} \frac{\mathrm{d} z_{1}}{\mathrm{~d} x}\right] \\
& =v_{1} u_{1}\left[k\left(y_{1}\right) a_{1} \frac{\mathrm{d} y_{1}}{\mathrm{~d} x}+K\left(z_{1}\right) b_{1} \frac{\mathrm{d} z_{1}}{\mathrm{~d} x}\right] .
\end{aligned}
\end{aligned}
$$

where $K\left(y_{1}\right)$ and $K\left(y_{2}\right)$ are the average number of mothers and aunts, respectively, of a juvenile male (e.g. $K\left(y_{1}\right)$ is the probability the mother of a juvenile male will be alive), and $a_{1}$ and $b_{1}$ are the rates at which a phenotypic change in a mother or an aunt affects juvenile male fitness. As in the transition from (2) to (3), to convert the phenotypic derivatives into relatedness coefficients, we divide by $\mathrm{d} y_{3} / \mathrm{d} x$. If, in addition, we divide by $u_{3}$, we get the first component of the inclusive fitness effect $\Delta W_{I F}$ in Table 2. Indeed, this follows from the observation that $\left(u_{1}\right)$ $\left.u_{3}\right) K\left(y_{1}\right)=m_{1}$, the average number of sons per mother, and $\left(u_{1} / u_{3}\right) K\left(z_{1}\right)=n_{1}$, the average number of nephews per aunt. To illustrate these last equations, if there were four juvenile males per adult female $\left(u_{1} / u_{3}=4\right)$ and a male had on average three aunts $\left(K\left(z_{1}\right)=3\right)$, then an adult female would have on average $4 \times 3=12$ nephews.

\section{EXAMPLE 3}

\section{Sex allocation}

Consider a sexual population in which $N$ mated females breed on a patch, with mating at random among the patch offspring, followed by partial dispersal of mated females at rate $\mu$, with $\operatorname{cost} c$, and then competition for the $N$ breeding sites among those mated females on each patch. This is the same patch 
structure as example 2 in which we found the ESS dispersal rate; here we want to calculate the ESS sex ratio of a mated female. For the special case of complete dispersal $(\mu=1)$, this problem was first studied by Hamilton (1967) in a classic paper on biased sex-ratios, and was then extended to haplodiploidy (Hamilton, 1979; Taylor \& Bulmer, 1980). For the case of partial dispersal, a one-locus genetic model was constructed by Bulmer (1986) and an inclusive fitness approach was presented by Frank (1986c) and Taylor (1988b).

In order to have a fixed population of offspring to deal with, it is technically convenient to model sex allocation in the following way: suppose each mother has exactly $n$ sons and daughters, but she gives each of her sons a survival probability $y$ and each of her daughters a survival probability $1-y$. Then $y$ functions as a sex ratio, measuring her investment in males.

The actor is the mother, and the two recipient classes are the male and female offspring, so what we need are expressions for the fitness of random female and male offspring in terms of the behaviour of the mother and her fellow breeders. The fitness of a random female offspring is

$$
\begin{array}{r}
W_{f}\left(y_{f}, z_{f}\right)=\left(1-y_{f}\right)\left[(1-\mu) p\left(z_{f}\right)\right. \\
\left.+\mu(1-c) p\left(y^{*}\right)\right]
\end{array}
$$

where $y_{f}$ is her mother's sex ratio, $z_{f}$ is the average sex ratio on her native patch, and $y^{*}$ is the average population-wide sex ratio. Here,

$p(z)=\frac{1}{n}\left[\frac{1}{(1-z)(1-\mu)+\left(1-y^{*}\right) \mu(1-c)}\right]$

is the breeding probability of a female who competes on a $z$-patch. Note that the normalized value of $p$ is

$$
p\left(y^{*}\right)=\frac{1}{n}\left[\frac{1}{\left(1-y^{*}\right)(1-c \mu)}\right]
$$

and it follows that the normal value of $W_{f}$ is $1 / n$. Similarly, the fitness of a random male offspring is

$$
\begin{aligned}
W_{m}\left(y_{m}, z_{m}\right) & =y_{m} \frac{1-z_{m}}{z_{m}} \\
\times & {\left[(1-\mu) p\left(z_{m}\right)+\mu(1-c) p\left(y^{*}\right)\right] }
\end{aligned}
$$

where $y_{m}$ is his mother's sex ratio, $z_{m}$ is the average sex ratio on his native patch, and the female:male mating ratio on his native patch is $\left(1-z_{m}\right) / z_{m}$. The normalized value of $W_{m}$ is $1 / n$, the same as $W_{f}$.
The average recipient fitness is

$$
W=c_{m} W_{m}+c_{f} W_{f}
$$

and the equilibrium equation $\mathrm{d} W / \mathrm{d} x=0$ evaluated at $y=x=y^{*}$ can be written

$$
F=M \frac{1-x^{*}}{x^{*}}
$$

with

$$
M=\left[c_{m} r_{m}-c_{m} R_{m}\right]
$$

and

$$
F=\left[c_{f} r_{f}+c_{m} R_{m}\right]-k^{2}\left[c_{f} R_{f}+c_{m} R_{m}\right] .
$$

where $k=(1-\mu) /(1-c \mu)$ is the probability that a mated female is native to her breeding patch (example 2 ), and the phenotypic derivatives $\mathrm{d} y_{j} / \mathrm{d} x$ and $\mathrm{d} z_{j} / \mathrm{d} x$ have been replaced by the relatedness coefficients $r_{j}$ and $R_{j}$, the relatedness of the mother to her own sex- $j$ offspring and to a patch offspring of $\operatorname{sex} j$ (including her own), respectively. This is the result that would be obtained with an inclusive fitness argument. Indeed, $F$ represents the valuation a mother puts on an extra daughter who breeds, and $M$ represents the valuation she puts on an extra son per successful mating with a breeding female; thus, the factor $\left(1-x^{*}\right) / x^{*}$ is the correction on male valuation for the number of mates per male. The first term in the expression for $F$ counts the daughter plus her mate - an extra female provides an extra mating for a male who might be related to the mother. The second term in $F$ accounts for the competitive effect of this extra daughter by subtracting the value of the displaced female, plus mate, weighted by the probability $k^{2}$ that two females are both native to their breeding patch. The first term in $M$ counts the extra son, and the second term accounts for the competitive effect of this extra son by subtracting the value of the displaced male. The reproductive values $c_{i}$ are obtained from the genetic system (diploid: $c_{f}=c_{m}$; haplodiploid: $c_{f}=2 c_{m}$; Price 1970), and the values of $r_{1}$ and $R_{1}$ are obtained from standard population genetic recursions, and depend on $N$ and $k$. Details for sex ratio models can be found in Frank (1986c) and Taylor (1988b).

Variations on this sex ratio model have been discussed by Frank (1986b,c, 1987) and Taylor (1988b). The advantage here is that the solutions for complex interactions among relatives follow directly and automatically from the fitness expression, $W$, and the algorithm of differentiation and replacement of derivatives by appropriate relatedness terms. Because these derivatives are the slopes of actor phenotype on recipient genotype, they are natural measures for the degree of shared reproductive interest measured by the kin selection coefficients. 


\section{DIFFERENT EFFECTS ON DIFFERENT COMPONENTS OF FITNESS}

Here we discuss how to handle a complication which often arises in age-structured models. Suppose the effect of an actor on a class- $j$ recipient is not to alter her overall fitness, but to modify different components of her fitness in different ways. For example, if the recipient is a mother, her production of daughters may be affected differently from her production of sons, or perhaps her fecundity is affected but not her survival. In this case, we treat these components as different classes of offspring, and modify the above class-structured analysis by writing her fitness $W_{j}$ in terms of these components, and looking at the effect of the action on each one. For example, an age-2 female who has five offspring surviving to next year and survives herself with probability $s$ to age 3 , would be regarded as dying and having five class- 1 offspring and $s$ class- 3 offspring.

We start by summarizing a few basic life history results (Charlesworth 1994) using the notation of Taylor (1990). Let $w_{i j}$ be the number of class $-i$ offspring of a class- $j$ individual. The count here is to be made according to genetic representation, that is if an individual furnishes only half the genes of an offspring, that offspring counts as a half. The offspring matrix

$$
\mathbf{A}=\left[w_{i j}\right]
$$

records in the $j$ th column the fitness components of a class $j$ individual. In an age-structured population, A is simply the Leslie matrix (Leslie, 1948). In a monomorphic population (uniform genic value $x^{*}$ ), the dominant eigenvalue $\lambda$ of $\mathbf{A}$ is the factor by which the population size is multiplied each generation. The vector $\mathbf{v}=\left(v_{i}\right)$ of individual reproductive values is in fact the dominant left eigenvector of $\mathbf{A}$ :

$$
\begin{gathered}
\lambda \mathbf{v}=\mathbf{v A} \\
\lambda v_{j}=\sum_{\mathrm{i}} v_{i} w_{i j}
\end{gathered}
$$

and the vector $\left[u_{i}\right]$ of equilibrium class frequencies is the dominant right eigenvector of A (Taylor, 1990):

$$
\begin{gathered}
\lambda \mathbf{u}=\mathbf{A u} \\
\lambda u_{i}=\sum_{j} w_{i j} u_{j} .
\end{gathered}
$$

The fitness of a class- $j$ individual is defined as the weighted sum:

$$
W_{j}=\sum_{i} \frac{v_{i}}{v_{j}} w_{i j}
$$

where the weight $v_{i} / v_{j}$ is the relative reproductive value of a class- $i$ offspring. Essentially the weights can be thought of as factors converting the fitness components into common units which can then be added. A comparison of (27) and (25) shows that, in a monomorphic population, the $W_{j}$ are all normalized to $\lambda$ (which is for most purposes the natural normalization), so that the conditions for (13) hold and the average fitness can be written [using (27)]:

$$
W=\sum_{j} c_{j} W_{j}=\sum_{i j} v_{i} w_{i j} u_{j}=\mathbf{v A u}
$$

where the last expression uses vector notation. If we allow mutant behaviour, the fitness components $w_{i j}$ become functions of $x$. We then proceed as in (14) and form the derivative

$$
\frac{\mathrm{d} W}{\mathrm{~d} x}=\sum_{i j} v_{i} \frac{\mathrm{d} w_{i j}}{\mathrm{~d} x} u_{j}=\mathbf{v} \frac{\mathrm{d} \mathbf{A}}{\mathrm{d} x} \mathbf{u} .
$$

As in (15), the differentiation treats the $v_{i}$ and the $u_{j}$ as constants (calculated in the monomorphic $x *$ population). This is discussed further in Appendix A. As in example 3, the relatednesses used in the derivative of $w_{i j}$ are those of the actor to the appropriate class- $j$ recipient.

\section{EXAMPLE 4}

\section{Offspring-parent altruism}

We consider a sexual population of hermaphrodites consisting of juveniles (class 1) and adults (class 2). Suppose mating is at random among the adults and the resulting offspring become juveniles the following year-each adult expecting $2 n$ offspring to survive to the juvenile stage, $n$ of its own (it is the "mother" of these) and $n$ through mating with other adults. Suppose that juveniles do not reproduce and they survive to become adults next year with probability $s$. In addition, adults of any age survive to the following year with probability $t$.

We have two classes with normal offspring matrix:

$$
\mathbf{A}=\left[\begin{array}{ll}
0 & n \\
s & t
\end{array}\right]
$$

Here each adult (col 2) gets half the genetic share of a total of $2 n$ offspring and survives with probability $t$. The dominant eigenvalue $\lambda$ of $\mathbf{A}$ is the solution of the characteristic equation

$$
\lambda^{2}-t \lambda-s n=0 .
$$

It is important to note that this is equivalent to the standard life history equation (Fisher, 1958, p. 26) which for this example would set the present value 
(discount rate $\lambda$ ) of all juvenile offspring of a juvenile to unity:

$$
1=\lambda^{-2} s n+\lambda^{-3} s t n+\lambda^{-4} s t^{2} n+\cdots=\frac{s n}{\lambda(\lambda-t)} .
$$

The $\lambda^{-2}$ in the first term accounts for the fact that the first set of offspring of a juvenile will be juveniles in 2 years' time.

It is easily checked that

$$
\mathbf{u} \propto[n, \lambda]^{\mathrm{T}}
$$

and

$$
\mathbf{v} \propto[s, \lambda]
$$

are the corresponding right and left eigenvectors for A, where " $\propto$ " indicates equality up to a multiplicative constant. Hence $(n, \lambda)$ and $(s, \lambda)$ are proportional to the vectors of class frequencies and individual reproductive values respectively. Note that $\mathbf{v}$ agrees with Fisher's (1958) definition of reproductive value as the total number of expected offspring discounted to present value, which for this example would give juvenile RV as $v_{1}=1$ [that's the sum in the middle of eqn (32)] and adult RV as $v_{2}=\lambda / s$. We look at altruistic behaviour of a juvenile towards its mother in case the mother has survived. Thus, the actor class is 1 and the recipient classes are 1 and 2 . The average fitness (13) is

$W=\sum c_{j} W_{j}=u_{1} v_{1} W_{1}+u_{2} v_{2} W_{2} \propto n s W_{1}+\lambda^{2} W_{2}$

using (12).

In order to highlight the role played by the fitness components, we present two similar examples here; in example 4a the interaction affects all components of individual fitness in the same way and the analysis can be done along the lines of example 3 , and in $4 \mathrm{~b}$, different components of fitness are affected differently, and we need the equations developed above.

\section{EXAMPLE 4A}

\section{All fitness components affected the same way}

We suppose that a single altruistic interaction increases the mother's entire fitness by a factor of $b$ but decreases the juvenile's fitness by a factor $c$. Let $y$ be the phenotype of a juvenile and let $z$ be the average phenotype of a mother's juvenile offspring. The fitnesses of the two different recipient classes (normalized to unity) are:

$$
\begin{gathered}
W_{1}=1-t c y \\
W_{2}=1+(t / \lambda) n b z
\end{gathered}
$$

where in (36) $t$ is the probability that the mother of the juvenile is alive, and in (37) $t / \lambda$ is the probability a random adult was an adult of the previous year (and in that case it can expect $n z$ altruistic interactions). If we form the average fitness (35), and take the derivative, we get

$$
\frac{\mathrm{d} W}{\mathrm{~d} x} \propto n t\left[-s c \frac{\mathrm{d} y}{\mathrm{~d} x}+\lambda b \frac{\mathrm{d} z}{\mathrm{~d} x}\right] .
$$

We replace $\mathrm{d} y / \mathrm{d} x$ by 1 and $\mathrm{d} z / \mathrm{d} x$ by the relatedness $R$ of the actor to its mother, and then the condition for the behaviour to be selected is that (38) be positive, which gives

$$
s c<\lambda b R \text {. }
$$

\section{EXAMPLE 4B}

\section{Different fitness components affected differently}

Now we suppose an altruistic interaction increases the mother's current fecundity by a factor of $b$ but does not affect her survival. In this case, we do the analysis with the matrix $\mathbf{A}$ which is now a function of $x$ :

$$
\mathbf{A}=\left[\begin{array}{cc}
0 & n(1+t n b z / \lambda) \\
s(1-t c y) & t
\end{array}\right]
$$

From (29), we form

$$
\begin{array}{r}
\frac{\mathrm{d} W}{\mathrm{~d} x}=\mathbf{v} \frac{\mathrm{d} \mathbf{A}}{\mathrm{d} x} \mathbf{u} \propto\left[{ }^{[s \lambda]}\left[\begin{array}{cc}
0 & n^{2} t b R / \lambda \\
-s t c & 0
\end{array}\right]\left[\begin{array}{l}
n \\
\lambda
\end{array}\right]\right. \\
=n s t[-\lambda c+b n R]
\end{array}
$$

where we have again set $\mathrm{d} y / \mathrm{d} x=1$, and $\mathrm{d} z / \mathrm{d} x=R$. The condition for the behaviour to be selected is that (41) be positive, which gives

$$
c<\frac{b n R}{\lambda}
$$

The critical cost-benefit ratio is proportional to the number of female offspring.

How is our intuition to compare the conditions (39) and (42) of examples $4 \mathrm{a}$ and $4 \mathrm{~b}$ for the spread of altruism? In (39) we are comparing fitness changes of two whole individuals, a juvenile with relative decrease $c$ (only survival) and an adult with relative increase $b$ (survival and fecundity), where we must use the weightings given by individual reproductive value, $s: \lambda$, and by relatedness to the juvenile actor, $1: R$. Condition (42) can be analysed in two different ways as presented in Table 3. Note that since the benefit is greater when the mother's whole fitness is increased, 
we expect (42) to imply (39). That will be the case if $n / \lambda<\lambda / s$ and this follows from the eigenvalue equation (31).

\section{Discussion}

Many inclusive fitness problems are of the simple, homogeneous kind of the first two examples. These models can be solved easily with our extension of the standard ESS technique: write a fitness function, maximize by differentiation, and solve for the optimum, using coefficients of relatedness in place of the phenotypic derivative.

Examples 3 and 4 are more challenging, and serve to demonstrate how the method incorporates reproductive values when individuals belong to different classes. In the sex allocation, example 3, it is not at all easy to come up with the expression (24) with a purely inclusive fitness analysis, so that the "automatic" construction of the derivative method is of considerable interest.

Example 4 is interesting in its own right, as there has been considerable discussion in the literature over whether inclusive fitness arguments can work in age-structured populations. For example, the controversy over parent-offspring conflict has been discussed by Alexander (1974), Parker (1985) and Clutton-Brock (1991). Charlesworth \& Charnov (1981) were perhaps the first to explain how inclusive fitness arguments should be constructed in this context; in particular they emphasized the role of

TABLE 3

The inclusive fitness calculation for example $4 b$

\begin{tabular}{lcccc}
\hline \multicolumn{4}{l}{ Method 1. Count "offspring" of recipients next year } \\
Recipient & Offspring & Class & RV & Relatedness \\
\hline mother & $2 n b$ & 1 & $s$ & $R / 2$ \\
juvenile & $-s c$ & 2 & $\lambda$ & 1 \\
$\Delta W_{I F}=2 n b s R / 2$ & $-s c \lambda=s(n b R-c \lambda)$ & &
\end{tabular}

Method 2. Measure fitness effects in terms of juveniles

\begin{tabular}{cccc} 
Recipient & $\begin{array}{c}\text { Juvenile } \\
\text { offspring }\end{array}$ & Time discount & Relatedness \\
\hline mother & $2 n b$ & $\lambda$ & $R / 2$ \\
juvenile & $-c$ & 1 & 1 \\
$\Delta W_{I F}=2 n b R / 2 \lambda-c=(n b R-c \lambda) / \lambda$ &
\end{tabular}

There are two different ways one might construct the inclusive fitness argument for example $4 \mathrm{~b}$. Method 1 follows the matrix approach of the text. The two components of fitness are treated as next year's "offspring," and the class of the offspring must be noted and weighted by the class RV. However, all these "offspring" belong to next year so that no weighting by time is required. Method 2 measures all fitness effects as "juvenile" (class 1) units, either this year or next year. Then RV weights are not required, but next year juveniles must be discounted by the population growth factor $\lambda$. Note that in each case the mother's juvenile offspring have relatedness to the actor of $R / 2$, assuming the mother's current mate is unrelated to the actor. reproductive value. One point of possible confusion in their treatment is the assertion (for example, in their abstract) that $\mathrm{RV}$ is relevant to effects on survival but not to effects on fecundity. They illustrate this with an example similar to our Example $4 \mathrm{~b}$ in which an actor suffers a loss of survival to increase the fecundity of a recipient, and the survival loss is weighted by $\mathrm{RV}$ but not the fecundity gain [Charlesworth \& Charnov, 1981, eqn (5a)]. In fact, what they are pointing out in their example is that the fecundity gain is not weighted by the RV of the recipient but it is weighted by the RV of the extra offspring that the fecundity gain represents. (Table 3, method 1). In the normalization of Charlesworth \& Charnov [1981, eqns (2) and (3)] the RV of a zygote is set equal to 1, and so it is a hidden weighting in the fecundity term. In this context, our example 4a is revealing, in that it shows that when the recipient has both components of fitness increased in the same way, then the appropriate weighting is indeed the recipient's RV. However, when different components of fitness are affected differently, as in example $4 \mathrm{~b}$, we measure the recipient's fitness through his components, regarded as different classes of "offspring," and the appropriate weighting is the RV of these classes [see the paragraph following eqn (42)]. Roger's (1993, Table 1) discussion of the evolution of menopause also makes this weighting explicit.

A typical objection to the inclusive fitness analysis of example 4 might claim that it underestimates the selective advantage of the altruistic behaviour in a juvenile because it does not not account for the advantage that it will accrue from its future altruistic offspring. We could go on like this - it also does not account for the disadvantage to future offspring, etc. Indeed, where is this argument to stop? As we have mentioned above, a valuable way to think about these models is to regard them as non-overlapping generation models, with all individuals alive in one year being "offspring" of those of the previous year. The question can now be asked in the general context of such models: when we are assessing the inclusive fitness effects of an action, do we take account of the possibility of altered behaviour in the next generation? For example, in sex allocation models, do we allow for the effects of the altered sex ratio in the female offspring when they become mothers? The answer is that we do not have to do that when selection is weak (ie. small selection differential), and this is supported by a number of analyses which demonstrate an equivalence between inclusive fitness and one-locus genetic models (Charlesworth, 1980; Michod \& Hamilton, 1980; Seger, 1981; Grafen, 1985; Taylor, 1989, 1996a). 
The theories of life history and kin selection have been developed to a high degree of sophistication. However, when faced with a particular biological problem, it is often difficult to put together the many demographic and social factors into a coherent analysis. Our method provides an orderly set of tools for studying the multiple pathways by which social interactions influence fitness. More importantly, the evolutionary processes stand out clearly during the analysis, so that the analysis itself enhances our understanding of the problem.

We are grateful to David Queller for a number of important comments which greatly clarified our logic and our exposition. P.D.T. is supported by the Natural Sciences and Engineering Research Council of Canada. S.A.F. is supported by NSF grant DEB-9057331 and NIH grant GM42403.

\section{REFERENCES}

Alexander, R. D. (1974). The evolution of social behavior. Ann. Rev. Ecol. Syst. 5, 325-383.

Bremermann, H. J. \& Pickering, J. (1983). A game-theoretical model of parasite virulence. J. theor. Biol. 100, 411-426.

Bresch, C., Niesert, U. \& Harnasch, D. (1980). Hypercycles, parasites and packages. J. theor. Biol. 85, 399-405.

Bulmer, M. G. (1986). Sex ratio theory in geographically structured populations. Heredity 56, 69-73.

Clutton-Brock, T. H. (1991). The Evolution of Parental Care. Princeton: Princeton University Press.

Charlesworth, B. (1980). Models of kin selection. In: Evolution of Social Behaviour: Hypotheses and Empirical Tests. (Mark, H., ed.), Weinheim: Verlag Chemie.

Charlesworth, B. (1994). Evolution in Age-Structure Populations, 2nd Edn. Cambridge: Cambridge University Press.

Charnov, E. L. (1977). An elementary treatment of the genetical theory of kin selection. J. theor. Biol. 66, 541-550.

Crow J. F. \& Kimura M. (1970). An Introduction to Population Genetics Theory. New York: Harper and Row.

FisHER, R. A. (1958). The Genetical Theory of Natural Selection, 2nd Edn. New York: Dover.

FranK, S. A. (1986a). Dispersal polymorphisms in subdivided populations. J. theor. Biol. 122, 303-309.

Frank, S. A. (1986b). Hierarchical selection theory and sex ratios. I. General solutions for structured populations. Theor. Pop. Biol. 29, 312-342.

Frank, S. A. (1986c). The genetic value of sons and daughters. Heredity 56, 351-354.

Frank, S. A. (1987). Variable sex ratio among colonies of ants. Behav. Ecol. Sociobiol. 20, 195-201.

Frank, S. A. (1994). Kin selection and virulence in the evolution of protocells and parasites. J. Proc. Royal Soc. Lond. B 258, $153-161$.

Grafen, A. (1985). A geometric view of relatedness. Oxford Surveys in Evolutionary Biology 2, 28-89.

Hamilton, W. D. (1964). The genetical evolution of social behaviour, I and II. J. theor. Biol. 7, 1-52.

Hamilton, W. D. (1967). Extraordinary sex ratios. Science 156, $477-488$.

Hamilton, W. D. (1972). Altruism and related phenomena, mainly in social insects. Ann. Rev. Ecol. Syst. 3, 192-232.

Hamilton, W. D. (1975). Innate social aptitudes of man: an approach from evolutionary biology. In: Biosocial Anthropology (Fox, R. ed.), pp. 133-155. New York: John Wiley and Sons.
Hamilton, W. D. (1979). Wingless and fighting males in fig wasps and other insects. In: Reproductive Competition and Sexual Selection in Insects (Blum, M. S. \& Blum, N. A. eds), New York: Academic Press.

Hamilton, W. D. \& May, R. M. (1977). Dispersal in stable habitats. Nature 269, 578-581.

LANDE, R. \& ARNOLD, J. S. (1993). The measurement of selection on correlated characters. Evolution 37, 1210-1226.

LeSLIE, P. H. (1948). Some further remarks on the use of matrices in population mathematics. Biometrika 35, 213-245.

Maynard Smith, J. (1979). Hypercycles and the origin of life. Nature 280, 445-446.

Maynard Smith, J. (1983). Models of evolution. J. Proc. Royal Soc. Lond. B 219, 315-325.

Maynard Smith, J. \& Price, G. R. (1973). The logic of animal conflict. Nature 246, 15-18.

Michod, R. E. \& Hamilton, W. D. (1980). Coefficients of relatedness in sociobiology. Nature 288, 694-697.

Orlove, M. J. \& Wood, C. L. (1978). Coefficients of relationship and coefficients of relatedness in kin selection: a covariance form for the rho formula. J. theor. Biol. 73, 679-686.

PARKER. G. A. (1985). Models of parent-offspring conflict. V. Effects of the behaviour of two parents. Animal Behaviour 3, 519-533.

PRICE. G. R. (1970). Selection and covariance. Nature 227, 520-521.

Queller, D. C. (1982). Kinship, reciprocity and synergism in the evolution of social behaviour. Nature 318, 366-367.

Queller, D. C. (1992). A general model for kin selection. Evolution 46, 376-380.

Rogers, A. R. (1993). Why menopause? Evol. Ecol. 7, 406-420.

SEgER, J. (1981). Kinship and covariance. J. theor. Biol. 91, 191-213.

Szathmary, E. \& Demeter, L. (1987). Group selection of early replicators and the origin of life. J. theor. Biol. 128, 463-486.

TAYLOR, P. D. (1988a). An inclusive fitness model for dispersal of offspring, J. theor. Biol. 130, 363-378.

TAYLOR. P. D. (1988b). Inclusive fitness models with two sexes. Theor. Pop. Biol. 34, 145-168.

TAYLOR, P. D. (1989). Evolutionary stability in one-parameter models under weak selection. Theor. Pop. Biol. 36, 125-143.

TAYLOR, P. D. (1990). Allele frequency change in a class-structured population. American Naturalist 135, 95-106.

TAYLOR, P. D. (1992). Altruism in viscous populations-an inclusive fitness model. Evol. Ecol. 6, 352-356.

TAYLOR P. D. (1996a). Inclusive fitness arguments in genetic models of behaviour. J. Math Bio. (in press).

TAYLOR, P. D. (1996b). The selection differential in quantitative genetics and ESS models. Evolution (in press).

TAYLOR, P. D. \& BULMER, M. G. (1980). Local mate competition and the sex ratio. $J$. theor. Biol. 86, 409-419.

\section{APPENDIX A}

When we differentiate eqns (14) and (28) with respect to $x$, an important simplification is our assumption that we can treat the $c_{j}, u_{j}$ and $v_{j}$ as independent of $x$, even though the deviant behaviour of a mutant allele will affect both its equilibrium distribution and its RV. In terms of eqn (28), when the entries of the matrix $\mathbf{A}$ become functions of $x$, so do its eigenvectors $\mathbf{v}$ and $\mathbf{u}$. In this case, the correct measure of fitness of the mutant gene, when rare, is not $W$ but is the dominant eigenvalue $\lambda=\lambda(x)$ of the matrix $\mathbf{A}=\mathbf{A}(x)$, as this is the rate of growth of the set of IBD copies of the allele when it is in equilibrium proportions among the classes. Now if we write 
$\mathbf{v}=\mathbf{v}(x)$ and $\mathbf{u}=\mathbf{u}(x)$ to display their dependence on $x$, then

$$
\mathbf{v}(x)[\mathbf{A}(x)-\lambda(x) \mathbf{I}] \mathbf{u}(x)=0
$$

where I denotes the identity matrix. Differentiate:

$$
\mathbf{v}^{\prime}[\mathbf{A}-\lambda \mathbf{I}] \mathbf{u}+\mathbf{v}\left[\mathbf{A}^{\prime}-\lambda^{\prime} \mathbf{I}\right] \mathbf{u}+\mathbf{v}[\mathbf{A}-\lambda \mathbf{I}] \mathbf{u}^{\prime}=0 .
$$

Since $\mathbf{u}$ and $\mathbf{v}$ are eigenvectors, the first and last terms, respectively, vanish giving:

$$
(\mathbf{v} \cdot \mathbf{u}) \lambda^{\prime}=\mathbf{v} \mathbf{A}^{\prime} \mathbf{u}=\frac{\mathrm{d} W}{\mathrm{~d} x}
$$

as in eqn (29), where everything is evaluated at $x=x^{*}$. Since $\mathbf{v}$ and $\mathbf{u}$ are positive, this shows that $\mathrm{d} \lambda / \mathrm{d} x$ and $\mathrm{d} W / \mathrm{d} x$ have the same sign. Thus, the equation $\mathrm{d} W / \mathrm{d} x=0$ gives the correct equilibrium condition.

\section{APPENDIX B}

A statistical analogue of eqn (1) is obtained by first regressing $W$ on the phenotypic values $y$ and $z$ :

$$
W=\beta_{W y . z} y+\beta_{W z, y} z+\delta
$$

where the $\beta$ are the partial regression coefficients, and $\delta$ is uncorrelated with $y$ and $z$. In our treatment, $W$ is a function of $y$ and $z$ (and this a common assumption of ESS models), but (B.1) is valid when
$W$ is only correlated with these variables. Then take the covariance with genic value $x$ :

$\operatorname{cov}(W, x)=\beta_{W y . z} \operatorname{cov}(y, x)+\beta_{W z . y} \operatorname{cov}(z, x)$

under the assumption that $\operatorname{cov}(\delta, x)=0$, which is to say that the only effect of the $x$-genotype on fitness is through the phenotypic values $y$ and $z$. This formulation is Queller's (1992) eqn (6). If we divide (B.2) by mean fitness, we get $\Delta \bar{x}$, the change in the mean of $x$ over one generation (Price, 1970).

If we divide eqn (B.2) by the variance of $x$, we get:

$$
\beta_{W x}=\beta_{W y . z} \beta_{y x}+\beta_{W z . y} \beta_{z x}
$$

and this is analogous to eqn (2).

The connection to eqn (2) is more striking if we assume that $y$ and $z$ are jointly normally distributed. In this case, a standard calculation (Lande \& Arnold, 1983; Taylor, 1996b) shows that the partial regression coefficients are equal to the expected value of the corresponding partial derivatives, and (B.3) becomes:

$$
\beta_{W x}=E\left(\frac{\partial W}{\partial y}\right) \beta_{y x}+E\left(\frac{\partial W}{\partial z}\right) \beta_{z x} .
$$

This suggests that the inclusive fitness formulation, eqn (3), should predict the change in $\Delta \bar{x}$ in a normally distributed population, if the derivatives of fitness with phenotype are replaced by their average values. 